Jennifer Patiño Aguilar 


\section{Aristóteles: crítica a la teoría de las ideas de Platón}

\section{Introducción}

En el presente ensayo se analiza la crítica que hace Aristóteles a la teoría de las ideas de Platón; comenzaremos por saber cómo y por qué Aristóteles critica la teoría de las ideas, después nos enfocaremos en el libro de la Metafísica de Aristóteles, de donde obtendremos las críticas que hace a esta teoría y, para finalizar, veremos qué respuestas da Aristóteles a varios de los problemas que existen en la teoría de las ideas de Platón. Al final llegaremos a unas conclusiones muy interesantes, porque notaremos que Aristóteles no estaba en contra de su maestro, sólo quería perfeccionar varias de sus ideas en su búsqueda de la verdad.

\section{¿Cómo y por qué Aristóteles critica la teoría de las ideas de Platón?}

Aristóteles, hijo del famoso médico del rey Filipo, preceptor, él mismo, del joven Alejandro, fue ya el que vio con claridad las flaquezas de que adolecía el pensamiento de Platón. En varios de sus escritos, con mucha frecuencia, Aristóteles polemiza contra Platón. No obstante, para Platón tiene Aristóteles los máximos respetos; en todo momento lo llama su maestro, su amigo. Y las objeciones que Aristóteles formula contra la teoría de las ideas de Platón se pueden reducir a seis grupos característicos.

En primer lugar, la duplicación innecesaria de las cosas. Aristóteles muestra que ese mundo de las ideas, que Platón construye metafísicamente con el objeto de "dar razón" de las cosas sensibles, es una duplicación del mundo de las cosas que resulta totalmente innecesaria. Esta objeción que hace aquí Aristóteles a Platón es de una importancia incalculable en el proceso del pensamiento filosófico griego, porque es la primera vez que la teoría de los dos mundos (el mundo sensible y el mundo inteligible) establecida por Parmé- 
nides dos siglos antes, la duplicidad de mundos, es insostenible. No hay un mundo inteligible de ideas contrapuesto y distinto del mundo sensible. Esto le parece una duplicación que no resuelve nada, porque sobre las ideas se plantearían exactamente los mismos problemas que se plantean sobre las cosas.

El segundo grupo de objeciones que Aristóteles hace a Platón es el de que el número de las ideas tiene que ser infinito, porque -dice Aristóteles- si dos cosas particulares, semejantes, son semejantes porque ambas participan de una misma idea (la participación es la "metaxis" de Platón), entonces, para advertir la semejanza entre una cosa y su idea hará falta una tercera idea; $y$ para advertir la semejanza entre esta tercera idea y la cosa, una cuarta idea y así infinitamente. De modo que la interposición de una idea para explicar la semejanza que existe entre dos cosas supone ya -implicaun número infinito de ideas.

El tercer argumento grave que Aristóteles formula contra Platón es el siguiente: que si hay ideas de cada cosa, tendrá que haber también ideas de las relaciones, puesto que las relaciones las percibimos intuitivamente entre las cosas.

A este argumento añade otro: que si hay ideas de lo positivo, de las cosas que son, tendrá que haber ideas de lo negativo, de las que no son, de las cosas que dejan de ser. Por ejemplo: si hay idea de la belleza, tendrá que haber idea de la fealdad; si hay idea del tamaño grande, tendrá que haber idea del tamaño pequeño, y en general, de cada tamaño. Pero los tamaños son infinitos: esto multiplicaría también innecesariamente el número de ideas.

La quinta objeción que Aristóteles formula es que la doctrina de las ideas no explica la producción, la génesis de las cosas. Las ideas en Platón son conceptos, definiciones hipostasiadas; pero esas definiciones hipostasiadas a lo más que pudieran llegar, si fuese inteligible la teoría de la participación, es a dar razón de lo que las cosas son, pero en ningún momento a explicar cómo las cosas advienen a ser. Esta introducción por Aristóteles de una exigencia de explicación para el advenir nos da una idea clara de que, por encima de la cabeza de Platón, quizá existió en Aristóteles una influencia profunda del viejo Heráclito, de aquel Heráclito que fijó su mirada preferentemente en lo que la realidad ofrece de mutable, de cambiable, de fluido.

Y la última, y quizá más importante objeción que Aristóteles opone a Platón, es la de que las ideas son trascendentes. El tras- 
cendentismo de las ideas le parece insostenible. No ve Aristóteles la necesidad de escindir y dividir entre las ideas y las cosas. Y precisamente esta objeción es importante porque la labor propia de Aristóteles en la filosofía se puede definir por un solo rasgo general con estas palabras: "Un esfuerzo titánico por traer las ideas platónicas del lugar celeste en que Platón las había puesto, y fundirlas dentro de la misma realidad sensible y de las cosas." Ese esfuerzo por deshacer la dualidad del mundo sensible y el mundo inteligible; por introducir en el mundo sensible la inteligibilidad; por fundir la idea intuida por la intuición intelectual con la cosa percibida por los sentidos, en una sola unidad existencial y consistencia; ese esfuerzo caracteriza supremamente la filosofía de Aristóteles, la metafísica de Aristóteles.

\section{Crítica a la teoría de las ideas en el libro de la Metafísica}

Aristóteles construyó un sistema filosófico propio. Previo a ello, sometió a crítica la teoría de las ideas de su maestro. Para intentar solventar las diferencias entre Heráclito y Parménides, Platón había propuesto la existencia de dos dimensiones en la realidad: la realidad sensible e inteligible. Para Aristóteles, el mundo no tiene compartimentos.

Si bien Aristóteles admite, al igual que Sócrates y Platón, que la esencia es lo que define al ser, no obstante (a diferencia de sus antecesores) concibe la esencia como la forma que está unida inseparablemente a la materia, constituyendo juntas el ser, que es la sustancia.

El Estagirita, en el Libro I de la Metafísica, propone que ésta consiste en el conocimiento de las causas y principios primeros. Las causas que propone el filósofo son cuatro: la causa formal, material, eficiente y final; y las justifica al demostrar que todos los filósofos anteriores a él ya habían mencionado estas mismas causas y no otras, aunque el tratamiento hacia éstas fue de una manera tosca y también confusa. Se propone, para demostrar esto, toda una amplia disquisición histórico-teórica.

Antes de llegar a la crítica realizada a la teoría platónica, Aristóteles pasa a exponer su teoría.

1 Abbagnano, Nicolás, Historia de la Filosofía, Vol. 1, ed. $1^{\circ}$ 1956, ed. $4^{\circ}$ 1994, Hora, Barcelona, p. 608. 


\section{"Todos los hombres tienen naturalmente el deseo de saber"2}

Aquí quiere demostrar que todos los hombres entienden por sabiduría el conocimiento de las causas y principios. Para mostrarnos lo comentado recientemente, hace una descripción de las diversas formas del conocer, que son la sensación, memoria, experiencia, arte y ciencia; procura indicar cómo se desarrollan unas a partir de otras y muestra cómo todos coinciden en tener por sabiduría solamente el arte y la ciencia:

En los hombres la experiencia proviene de la memoria. Pero la experiencia, al parecer, se asimila casi a la ciencia y al arte. Por la experiencia progresan la ciencia y el arte en el hombre. ${ }^{3}$

El arte comienza, cuando de un gran número de nociones suministradas por la experiencia, se forma una sola concepción general que se aplica a todos los casos semejantes. ${ }^{4}$

Esto consiste en que la experiencia es el conocimiento de las cosas particulares, y el arte, por lo contrario, el de lo general. ${ }^{5}$

La experiencia se refiere, entonces, siempre a lo particular, mientras el arte y la ciencia apuntan a lo universal, es decir, al porqué de las cosas, a su causa. El sabio es tenido por tal en la medida en que se eleva a un saber más allá de las necesidades prácticas, y este saber no es otro que el del conocimiento puro de las causas. El sabio, entonces, es aquel que lo sabe todo, sin poseer la ciencia particular de ninguna cosa, posee la ciencia universal en sumo grado. Para El Estagirita, la sabiduría, la ciencia más plena, es aquella que versa sobre el estudio de los primeros principios y de las causas, es decir, la filosofía.

A continuación pasa a estudiar las respuestas de los antiguos a la búsqueda de las causas, siguiendo las diferentes corrientes, que trataré de exponer brevemente: Tales de Mileto, al ver que el alimento es húmedo y que el calor nace de lo húmedo, afirma que

2 Aristóteles, Metafísica, Gredos, Madrid (Libro I, Metafísica Cap. I), 1994, p. 69.

3 Aristóteles, Metafísica, Gredos, Madrid (Libro I, Metafísica Cap. I). 1994, p.70.

4 Ibidem p. 71.

5 Ibidem p. 72.

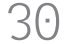


el agua es el principio buscado; por su parte, Anaxímenes propone que el primer principio es el aire; siguiendo con la exposición aparece Empédocles, el cual toma los dos anteriores, junto con la tierra, como principios del mismo rango. Anaxágoras dice que hay infinitos principios que se juntan y se separan para generar o destruir las cosas.

[...] los más antiguos admiten un principio corporal, porque el agua y el fuego y las cosas análogas son cuerpos; en los unos, este principio corporal es único, y en los otros múltiple; pero unos y otros lo consideran desde el punto de vista de la materia. ${ }^{6}$

Todos estos filósofos dieron respuesta a la pregunta de la causa material de las cosas, y Aristóteles se pregunta entonces por la causa de que estos elementos se unan o separen para generar o destruir las cosas: la causa del movimiento o causa efectiva.

Los pitagóricos, ciertamente, han hablado también de dos principios. El finito, el infinito y la unidad, no son según ellos, naturalezas aparte, como lo son el fuego o la tierra [...], sino que el infinito en sí y la unidad en sí son la sustancia misma de las cosas [...], y por consiguiente, el número es la sustancia de todas la cosas. ${ }^{7}$

$\mathrm{Al}$ ver que todo se rige según números matemáticos y armonías, pensaron que el primer principio de las cosas era el mismo que el de las matemáticas, o sea, los números. Lo que no cuadraba en su sistema lo arreglaban con invenciones. El uno sería el primer elemento, y luego habría un montón de parejas de opuestos, que son los principios de los entes. En todos los casos, los elementos que constituyen la materia son inmanentes a ella.

Como recapitulación de todo lo anterior, Aristóteles plantea una conclusión: sólo se tratan la causa material y la eficiente, y los principios pueden ser uno o varios, pero siempre de índole material.

En el sexto capítulo aparece ya Platón: "A estas diversas filosofías siguió la de Platón..."

6 Aristóteles, Metafísica, Gredos, Madrid (Libro I, Metafísica Cap. V). 1994, p. 77.

7 Ibidem p. 81. 
A continuación expondré las críticas referidas a la teoría de las ideas de Platón por parte de Aristóteles.

La Metafísica aristotélica se elabora en buena medida como reacción a la teoría de la ideas de Platón, pero no tiene sentido buscar en la crítica del Estagirita a dicha teoría ningún tipo de razón personal que pudiera haber enfrentado a Aristóteles con Platón, sino, como el mismo autor de la Metafísica expone en su obra, se trata de la simple búsqueda de la verdad. Existe entre ambos un punto común entre sus teorías, y se basa en la existencia de un elemento común entre todos los objetos de la misma clase, el universal, la idea, que es la causa de que apliquemos la misma denominación a todos los objetos del mismo género; admitirá, por lo tanto, que ese universal es real, pero no que tenga existencia propia, independiente de las cosas; según Aristóteles, Platón duplica, sin motivo, el mundo de las cosas visibles, estableciendo una realidad paralela que necesitará, a su vez, de explicación.

Platón [...] creyó que sus definiciones debían recaer sobre otros seres que los seres sensibles, porque ¿cómo dar una definición común de los objetos sensibles que mudan continuamente? Estos seres los llamó Ideas, añadiendo que los objetos sensibles están fuera de las Ideas, y reciben de ellas su nombre, porque en virtud de su participación en las Ideas, todos los objetos de un mismo género reciben el mismo nombre que las Ideas. La única mudanza que introdujo en la ciencia fue esta palabra, participación. Los pitagóricos dicen, en efecto, que los seres existen a imitación de los números...

Tampoco es capaz de explicar el movimiento de las cosas, que era uno de los motivos de su formulación; ahora bien, esta teoría no ofrece ningún elemento para explicar el movimiento, el cambio, ya que siendo las ideas inmóviles e inmutables, si las cosas son una imitación de las ideas, habrían de ser también inmóviles e inmutables; pero si cambian, ¿de dónde proceden?

Estos filósofos solo se han fijado en la causa material [... ] Los que mejor lo han hecho son los que han recurrido a las ideas y a

8 Aristóteles, Metafísica, Gredos, Madrid (Libro I, Metafísica Cap. VII), 1994. 
los elementos de las ideas; porque no consideran las ideas y sus elementos, ni como la materia de los objetos sensibles, ni como los principios del movimiento. Las ideas, según ellos, son más bien causas de inmovilidad y de inercia [..+ $]$ En cuanto a la causa final de los actos, de los cambios, de los movimientos, nos hablan de alguna causa de este género, pero no le dan el mismo nombre que nosotros ni dicen en qué consisten. Los que admiten como principios la inteligencia o la amistad, dan a la verdad estos principios como una cosa buena, pero no sostienen que sean la causa final de la existencia o de la producción de ningún ser, y antes dicen, por lo contrario, que son las causas de sus movimientos."

Aristóteles considera que la teoría de las ideas es imposible, ya que establece una separación entre el grado de realidad visible y el inteligible; dicho de otro modo, entre la sustancia y aquello por lo que una sustancia es, su forma o esencia. Las ideas, en efecto, representan la esencia de las cosas, es decir, aquello por lo que las cosas son lo que son.

Preguntas del tipo ¿cómo es posible que aquello por lo que algo es lo que es no resida en el objeto, sino fuera de él?, ¿cómo es posible que aquello que hace que el hombre sea hombre, su esencia, la idea de hombre, no resida en el hombre, sino que exista independientemente de él?, hacen que las formulaciones realizadas por Platón para tratar de explicar la relación entre las ideas y las cosas, las teorías de la participación y la imitación, lejos de explicar dicha relación, no sean más que metáforas.

Ya que el mismo Platón había criticado dichas teorías en el Parménides; Aristóteles insistirá en sus carencias con el argumento del "tercer hombre"; si el hombre es el resultado de la imitación de la idea de hombre, y tal idea es entendida como una entidad de carácter individual, ¿a qué otra realidad imita la idea de hombre? Debe existir un tercer modelo de hombre para explicar la similitud entre los hombres concretos. De ese modo encadenaríamos hasta el infinito la exigencia de un modelo del modelo, lo que nos llevaría al absurdo. Por otra parte, las cosas no pueden provenir de la ideas; sin embargo, ésa es una afirmación crucial de la teoría de las ideas, al concebir que éstas son causa de las cosas; no obstante, es el mismo

9 Aristóteles, Metafisica, Gredos, Madrid (Libro I, Metafísica Cap. VII), 1994. 
Platón quien explica que las ideas vienen a ser las causas ejemplares de las cosas, pero no sus causas eficientes.

En esta crítica aristotélica a la teoría de la ideas se vislumbran ya los fundamentos de su propia metafísica; ante la imposibilidad de que las ideas expliquen coherentemente la causa de lo real, propondrá la teoría de las cuatro causas del ser, y ante la irrealidad de las ideas, propondrá su teoría de la sustancia. La inconsistencia de la explicación platónica del cambio, por lo demás, le llevará a proponer la distinción entre ser en acto y ser en potencia.

\section{Propuestas de Aristóteles}

\section{Substancia, esencia, accidente}

El propósito de Aristóteles es, primeramente, traer las ideas trascendentes de Platón y fundirlas con las cosas reales de nuestra experiencia sensible. Para ello comienza partiendo de la cosa tal como la vemos y sentimos. Y en la cosa real, tal como la vemos y sentimos, distingue Aristóteles tres elementos: un primer elemento que llama substancia, un segundo elemento que llama esencia y un tercer elemento que llama accidente.

¿Qué es la substancia? La palabra tiene en Aristóteles dos significaciones. Aristóteles la emplea indistintamente en una y otra significación. Unas veces - la mayor parte - tiene un primer sentido estricto; otras, uno lato. El sentido estricto es el de la unidad, que soporta todos los demás caracteres de la cosa. Si nosotros analizamos una cosa, descubrimos en ella caracteres, notas distintivas, elementos conceptuales: este vaso es grande, es de cristal, es frío, tiene agua dentro, ha sido hecho de esta manera o de esta otra, pero el quid, del cual se dice que es esto, que es lo otro, que ha sido hecho de esta manera o de la otra manera; el quid, como dice Santo Tomás, la "quiddítas", la cosa de la cual se predica todo lo que se puede predicar, eso lo llama Aristóteles el "substante", en griego "hípojéimenos", que yace debajo, que los latinos han traducido por la palabra "substare", estar debajo'; lo llama la "substancia". La substancia es, en suma, el correlato objetivo del sujeto en la proposición, del sujeto en el juicio.

Pero, ¿qué decimos de la substancia? Pues todo lo que decimos de la substancia es lo que llama Aristóteles esencia. La esencia es la suma de los predicados que podemos predicar de la substan- 
cia. Ahora, estos predicados se dividen en dos grupos: predicados que convienen a la substancia de tal suerte que si le faltara uno de ellos no sería lo que es, y luego predicados que convienen a la substancia, pero que son de tal suerte que aunque alguno de ellos faltara, seguiría siendo la substancia lo que es. Aquellos primeros son la esencia propiamente dicha, porque si alguno de ellos le faltara a la substancia, la substancia ya no sería lo que es, y estos segundos son el accidente, porque el hecho de que los tenga o no los tenga, no entorpece para nada a que sea lo que es. ${ }^{10}$

De esta manera llegamos al otro sentido que de vez en cuando da Aristóteles a la palabra substancia, y es el sentido de la totalidad de la cosa, con sus caracteres esenciales y accidentales. En ese sentido llama Aristóteles la substancia, lo individual. Por eso, para Aristóteles la respuesta a la pregunta de la que han arrancado estas conferencias, estas excursiones por la metafísica, es muy simple y está completamente de acuerdo con la propensión natural del hombre. La respuesta a la pregunta: ¿quién existe?, es para Aristóteles ésta: existen las cosas individuales; lo demás no existe, son substancias "secundas" "dentere usía", substancias segundas, que no tienen más existencia secundaria, el ser que consiste en ser predicado o predicable, pero nada más. ${ }^{11}$

\section{La materia y la forma}

Pero no se contenta Aristóteles con traer las ideas del cielo a la Tierra. Recuerden ustedes que una de las críticas fundamentales que él hace a Platón consiste en reprocharle que las ideas no tienen "actuación", no actúan, son inoperantes, no tienen fuerza genética y generadora. Aristóteles, al traer las ideas al mundo de las cosas, quiere darle fuerza genética y generadora. Por eso establece en cada cosa una distinción fundamental. Lo mismo que en el análisis de la cosa distingue la substancia, la esencia y el accidente, distingue ahora en la cosa estos dos elementos: la forma y la materia.

¿A qué llama Aristóteles materia? Aristóteles llama materia a un concepto que no tiene nada que ver con lo que en física llamamos nosotros hoy materia. Materia, para él, es simplemente aque-

10 Copleston, Frederick, Historia de la Filosofía, Grecia y Roma, Tomo I, Liber, 1983, Madrid.

11 Ibidem. 
llo con que está hecho algo. El "aquello con que está hecho algo"12 puede ser eso que nuestros físicos hoy llaman materia; pero puede ser también otra cosa que no sea eso que los físicos hoy llaman materia. Así, una tragedia es una cosa que ha hecho Esquilo o que ha hecho Eurípides, y esa cosa está hecha con palabras, con "logoi", con razones, con dichos de los hombres, con sentimientos humanos; y no está hecha con materia en el sentido que dan a la palabra materia los físicos de hoy. Materia es, pues, para Aristóteles aquello -sea lo que fuere- con que algo está hecho.

¿Y forma? ¿Qué significa forma para Aristóteles? Ésta es una de las palabras que más ha dado quehacer a los filósofos e historiadores de la filosofía. No niego yo que sea difícil interpretar lo que Aristóteles quiso llamar "forma". No niego que sea difícil. Tampoco niego que la interpretación que yo les doy a ustedes no esté expuesta a toda suerte de crítica. No hay una sola de las interpretaciones que se han dado de la "forma" en Aristóteles que no esté expuesta a toda suerte de críticas. Pero yo, que no voy a entrar ahora en polémica con todas y cada una de las acepciones que esta palabra ha tenido y tiene, me voy a contentar con dar mi interpretación.

La palabra forma la toma Aristóteles de la geometría; de la influencia que la geometría tiene sobre Sócrates y sobre Platón. No olviden ustedes que Platón inscribió en la puerta de su escuela, que se llamaba la Academia, un letrero que decía: "Nadie entre aquí si no es geómetra". Aristóteles entendió por forma, primero y principalmente, la figura de los cuerpos, la forma como terminación límite de la realidad corpórea vista desde todos los puntos de vista; la forma en el sentido de la estatuaria, en el sentido de la escultura; eso entendió primero y fundamentalmente por forma Aristóteles. Pero sobre esa acepción y sentido de la palabra forma, entendió Aristóteles también aquello que hace que la cosa sea lo que es, aquello que reúne los elementos materiales, en el sentido amplio que les dije a ustedes antes, entrando también lo inmaterial. Aquello que hace entrar a los elementos materiales en un conjunto, que les confiere unidad y sentido, eso es lo que llama Aristóteles forma. La forma, pues, se confunde con el conjunto de los caracteres esenciales que hacen que las cosas sean lo que son; se confunde con la esencia. La forma, en Aristóteles, es la esencia, lo que hace que la cosa sea lo que es.

12 Idem. 
Ahora bien: esas formas de las cosas no son para Aristóteles formas azarosas, no son formas casuales, no han sido traídas por el ir y venir de las causas eficientes en la naturaleza. Lejos del pensamiento de Aristóteles, lo más lejos posible, está nuestra idea de física moderna de que lo que cada cosa físicamente es, sea el resultado de una serie de causas puramente físicas, eficientes, mecánicas, que sucediéndose unas a otras han venido necesariamente a parar a lo que una cosa en este momento es. Nada hay más lejos del pensamiento aristotélico que eso; sino que para Aristóteles cada cosa tiene la forma que debe tener, es decir, la forma que define la cosa. Por consiguiente, para Aristóteles la forma de algo es lo que a ese algo le da un sentido; y ese sentido es la finalidad, es el "telos", palabra griega que significa 'fin': de ahí viene esta palabra que se usa mucho en filosofía y que es teleología, 'teoría de los fines', el punto de vista desde el cual apreciamos y definimos las cosas, no en cuanto que son causas mecánicamente, sino en cuanto que están dispuestas para la realización de un fin. Pues bien: para Aristóteles la definición de una cosa contiene su finalidad, y la forma o conjunto de las notas esenciales imprime en esa cosa un sentido que es aquello para lo que sirve.

De esta manera está ya armado Aristóteles para contestar a la pregunta acerca de la génesis o producción de las cosas. Si la materia y la forma son los ingredientes necesarios para el advenimiento de la cosa, entonces ese advenimiento, ¿’n qué consiste? Consiste en que a la materia sin forma, se añade, se agrega, se sintetiza con ella la forma. Y la forma, ¿qué es? La forma es la serie de las notas esenciales que hacen de la cosa lo que es y le dan sentido y telos, finalidad.

\section{Conclusiones}

¿Cómo y por qué Aristóteles critica la teoría de las ideas de Platón?

Realmente Aristóteles no intenta ir en contra de su maestro Platón, ya que él tiene muchas bases del pensamiento de éste que se ven en tres aspectos: primero, que el ser de las cosas sensibles es problemático. Necesitará Aristóteles explicar en qué sentido y cómo las cosas sensibles son. El punto de partida seguirá siendo, para Aristóteles, lo mismo que para Platón y para Parménides, que los sentidos, el espectáculo abigarrado del mundo con sus varia- 
dos matices, no es el verdadero ser, sino que es un ser puesto en interrogante; es un ser problemático que necesita una explicación. Segundo: la explicación del ser problemático de las cosas sensibles consistirá en descubrir detrás de ellas lo intemporal y lo eterno. Aristóteles mostrará contra el movimiento, contra la temporalidad, la misma antipatía que Parménides, Zenón y Platón. Tercero: que Aristóteles, aunque percibe muy bien el flaco de Parménides y el flaco de Platón -que han consistido en confundir constantemente, o mejor dicho en fundir constantemente la esencia y la existenciaseguirá él mismo también cometiendo ese error. Lo cometerá en otra forma completamente distinta: afirmando una distinción conceptual entre ellas, pero seguirá estableciendo una función o distinción real entre la esencia y la existencia.

\section{Crítica a la teoría de las ideas en el libro de la Metafísica}

La metafísica aristotélica se elabora en buena medida como reacción a la teoría de las ideas de Platón. No parece que Aristóteles haya manifestado ninguna oposición crítica a la teoría de las ideas durante su permanencia en la Academia. Todo indica, por el contrario, que las primeras críticas a la teoría de las ideas se elaboran luego de su abandono de la Academia, cuando Aristóteles comienza a perfilar su propia filosofía. Hay que recordar, sin embargo, que ya Platón había criticado la teoría de las ideas en el Parménides, y que probablemente la teoría de las ideas había sido objeto de numerosas controversias en la Academia. No tiene sentido, pues, buscar en la crítica aristotélica a la teoría de las ideas ningún tipo de razón personal que pudiera haber enfrentado a Aristóteles con Platón, sino, como el mismo Aristóteles nos dice en la Metafísica, la simple búsqueda de la verdad.

Aristóteles estará de acuerdo con Platón en que hay un elemento común entre todos los objetos de la misma clase, el universal, la idea, que es la causa de que apliquemos la misma denominación a todos los objetos del mismo género; admitirá, por lo tanto, que ese universal es real, pero no que tenga existencia independiente de las cosas, es decir, que sea subsistente. La teoría de las ideas, por lo demás, al dotar de realidad subsistente al universal, a la idea, duplica sin motivo el mundo de las cosas visibles, estableciendo un mundo paralelo que necesitaría a su vez de explicación. 


\section{Propuestas de Aristóteles}

\section{Substancia, esencia, accidente}

Ustedes ven aquí lo que ha hecho Aristóteles, la faena magnífica que ha llevado a cabo. Ha consistido esta faena en aislar el elemento existencial que hay en el parmenismo y colocarlo como "hipojéimenos", como "substancia", en el sentido estricto de la palabra; en tomar luego la idea platónica, que era la unidad puramente esencial de los caracteres de la definición, del "logos" de Sócrates, del concepto y atribuirla a la substancia, como lo que designa lo que la substancia es, y añadir luego los caracteres particulares que la experiencia nos muestra en cada una de las substancias.

Ha logrado Aristóteles magníficamente lo que se proponía: traer las ideas del cielo a la Tierra; destruir la dualidad del mundo sensible y el inteligible; fundir estos dos mundos en el concepto lato de la substancia, de cosa real, que está ahí. En este mundo sensible cada cosa es, existe, tiene una existencia, es una substancia. Pero, ¿qué es lo que eso es?, ¿̇en qué consiste eso que es? Viene inmediatamente el concepto, la idea platónica, que desciende de su mundo hiperbóreo y viene a posarse sobre la realidad existencial de la substancia para darle la posibilidad de una definición, para hacerla inteligible, para que el pensamiento pueda pensarla, definirla, fijarla en el catálogo general de los seres; y luego los elementos no esenciales, accidentales, que no añaden ni quitan a la definición esencial, pero que caracterizan la substancia como esto que está en este lugar y en este momento.

\section{La materia y la forma}

Ahora bien: ¿qué es esto, sino la idea platónica que vimos descender del cielo para posarse sobre la substancia y formar la totalidad e integridad de la cosa real? Pues a esa idea platónica no le da Aristóteles tan sólo, como hacía Platón, la función de definir la cosa sino que es posible ver aquí ahora que le da función de lograr el advenimiento de la cosa. La cosa adviene a ser lo que es porque su materia es informada, es plasmada, recibe forma, y una forma que es la que le da sentido y finalidad. Pero esto da a las ideas platónicas lo que las ideas platónicas no tienen; imprime una capacidad dinámica, una capacidad productiva a las ideas traídas aquí 
al mundo sensible en la figura de forma y bajo el aspecto de forma. En esas ideas está para Aristóteles el germen, el principio informativo, creador, productivo, de la realidad de cada cosa.

\section{Epílogo}

¿Qué implica esto? Implica evidentemente algo que ya sale por completo de los límites en que se movía la filosofía de Platón, porque implica, sin que haya de ello la menor duda, que cada cosa es lo que es porque ha sido hecha inteligentemente. Si la forma de la cosa es lo que confiere a la cosa su inteligibilidad, su sentido, su telos, su fin, no hay más remedio que admitir que cada cosa ha sido hecha del mismo modo como el escultor hace la estatua, como el carpintero hace la mesa, como el herrero hace la herradura. Han tenido que ser hechas todas las cosas en el universo, todas las realidades existenciales por una causa inteligente, que ha pensado el telos, la forma, y que ha impreso la forma, el fin, la esencia definitoria en la materia. 
